\title{
Diagnóstico diferencial de tumores de glândulas salivares maiores
} Differential diagnosis of major salivary gland tumors

\author{
Lucas Bozzetti Pigozzi \\ Rafael Morawski* \\ João Batista Burzlaff ${ }^{* *}$ \\ Leonardo Tonietto**** \\ Vinícius Salim Silveira ${ }^{* * * *}$ \\ Thiago Calcagnotto ${ }^{* * * * * *}$
}

\section{Resumo}

Objetivo: o objetivo deste estudo é abordar e discutir, por meio de um relato de caso, aspectos clínicos e imaginológicos capazes de estabelecer o diagnóstico diferencial das lesões tumorais de glândulas salivares maiores. Relato de caso: o presente estudo discute, com um relato de caso clínico, o diagnóstico diferencial de glândulas salivares maiores em que um paciente relatou um aumento de volume, com diagnóstico médico inicial de linfadenopatia cervical crônica causada por histórico de infecções recorrentes na garganta. Sendo assim, a conduta inicial realizada por um médico-cirurgião de cabeça e pescoço foi a preservação do caso. Porém, após nova reavaliação e exames complementares solicitados pela equipe de cirurgia bucomaxilofacial devido à insistência dos familiares, foi cogitada a hipótese diagnóstica de adenoma pleomórfico de glândula submandibular. Em consequência, foi realizada a cirurgia para acesso e remoção da lesão, e esta foi enviada para diagnóstico histopatológico, que permitiu concluir que se tratava de adenocarcinoma de glândula submandibular. Considerações finais: o diagnóstico diferencial é fundamental na determinação da escolha de tratamento dessas neoplasias, uma vez que, além da conduta terapêutica diferente, as lesões malignas podem gerar metástases em outras partes do corpo, tornando o prognóstico de sobrevida do paciente menos positivo. $O$ correto diagnóstico, associado a uma terapêutica adequada e, principalmente, ao diagnóstico precoce do tipo de lesão, propicia uma melhor qualidade de vida ao paciente acometido por uma neoplasia maligna de glândula salivar maior.

Palavras-chave: Glândulas salivares. Diagnóstico diferencial. Adenocarcinoma.

\section{Introdução}

O corpo humano é composto por glândulas salivares maiores e menores, sendo as maiores as glândulas parótidas, as submandibulares e as sublinguais. Ambas podem ser acometidas por diferentes tipos de patologias, entre benignas e malignas ${ }^{1,2}$. As patologias malignas que acometem glândulas salivares maiores são consideradas raras e têm como exemplos mais comuns o carcinoma mucoepidermoide, adenocarcionomas e o tumor misto maligno $^{3,4}$. Essas neoplasias representam $20 \%$ dos casos de todas as lesões de glândulas salivares ${ }^{5}$; a parótida é a glândula mais acometida por tumores de glândulas salivares $(70,6 \%)$, seguida da glândula submandibular (19,3\%), das glândulas salivares menores $(9,24 \%)$ e, mais raramente, das glândulas sublinguais $(0,8 \%)^{6}$.

As neoplasias malignas de glândula salivar são principalmente caracterizadas clinicamente por uma taxa de crescimento rápido e adenopatia cervical $^{7}$.

Entre as neoplasias, os adenocarcinomas são lesões malignas que devem ser diagnosticadas precocemente, pois são de difícil diagnóstico clínico e histopatológico ${ }^{8}$. Devido isso, com o tempo, podem fazer metástase para linfonodos regionais em cerca de $16 \%$ dos pacientes, principalmente em idade entre 40 e 70 anos, apresentando um diagnóstico desfavorável, com sobrevida de aproximadamente 7 meses $^{8-11}$. O adenocarcinoma pode afetar tanto glândulas salivares maiores quanto menores, porém

\section{http://dx.doi.org/10.5335/rfo.v22i2.7294}

\footnotetext{
Cirurgião-dentista graduado na Universidade Federal do Rio Grande do Sul (Ufrgs).

Cirurgião-dentista graduado na Ufrgs.

Doutor em Patologia pela Ufrgs, mestre em CTBMF pela PUCRS, professor adjunto da Faculdade de Odontologia da Ufrgs, professor do Curso de Especialização em CTBMF, Fatec Dental Ceeo.

Doutor em CTBMF pela PUCRS, professor do Curso de Especialização em CTBMF, Fatec Dental Ceeo.

Doutor em CTBMF pela PUCRS, professor do Curso de Especialização em CTBMF, Fatec Dental Ceeo.

Mestre em CTBMF pela Ufrgs, coordenador do Curso de Especialização em CTBMF, Fatec Dental Ceeo.
} 
apresenta uma taxa de sobrevida maior, quando acomete glândulas salivares menores ${ }^{8,12}$.

Para o diagnóstico de lesões de glândulas salivares, é necessário inicialmente a realização de um exame clínico, seguido, se necessário, de exames imaginológicos, biópsia, exames histopatológicos, entre outros. Deve-se estar atento ao aumento de volume que pode ser compatível com alguma linfoadenopatia, porém é necessário acompanhamento quando há essa dúvida (linfoadenopatia ou lesão de glândulas salivares), para que não ocorra omissão frente a uma lesão maligna e de origem glandular com risco de metástase ${ }^{13-15}$.

Para isso, muitas vezes é necessário a realização de diagnóstico diferencial, a fim de eliminar outras condições semelhantes que possam confundir o profissional na tomada de decisão clínica. Portanto, este trabalho tem como objetivo abordar e discutir, por meio do relato de caso, aspectos clínicos capazes de estabelecer o diagnóstico diferencial das lesões tumorais de glândulas salivares maiores.

\section{Relato de caso}

Paciente do gênero feminino, leucoderma, com 45 anos de idade e sem histórico de alterações sistêmicas, de hábito tabagista ou etilista, procurou atendimento para avaliação de lesão nodular com pequeno aumento de volume em região cervical esquerda, cerca de $10 \mathrm{~mm}$ à frente do ângulo mandibular. A paciente relatava conhecimento de aumento e volume endurecido há cerca de dois anos e possuía diagnóstico, dado por especialista em cirurgia de cabeça e pescoço, na época, de linfoadenopatia cervical crônica causada por histórico de infecções recorrentes na garganta, por isso sem necessidade de intervenção e com orientação de aguardar a involução do quadro. O exame de ressonância magnética, também da época, evidenciou lesão nodular com hipersinal em região cervical esquerda e adjacente à glândula submandibular esquerda, apresentando limites definidos.

A consulta com cirurgião bucomaxilofacial ocorreu em função da insistência dos familiares da paciente, a fim de que ela buscasse uma segunda opinião em função de observar a manutenção do quadro clínico, mesmo transcorridos dois anos em relação ao primeiro diagnóstico e sem queixas da paciente associadas ao problema. $\mathrm{O}$ quadro clínico relatado pela paciente ao cirurgião bucomaxilofacial foi o mesmo de há dois anos em termos de manutenção da lesão: pouca mobilidade e dor ao toque e à pressão mais intensa. Os exames radiográficos em norma frontal e teleperfil, solicitados pela cirurgiã-dentista clínica geral da paciente, não evidenciaram achados radiográficos anormais. Baseado nos achados clínicos relatados pela paciente e observados em consulta, associados às imagens da ressonância magnética solicitadas pelo cirurgião bucomaxilofacial, que evidenciaram uma imagem hiperdensa, adjacente e em continuação à glândula submandibular esquerda, conforme visto na Figura 1, o diagnóstico clínico foi de adenoma pleomórfico de glândula submandibular. A partir disso, estabeleceu-se como conduta a realização de cirurgia para acesso e remoção da lesão.

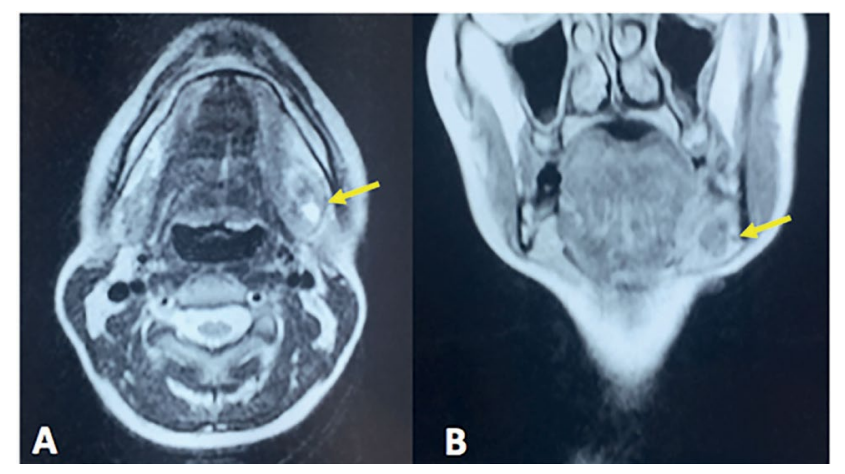

Figura 1 - Exame de ressonância magnética em cortes axiais (A) e coronais (B)

Legenda: as setas amarelas destacam, em ambos os cortes, lesão de aspecto lobular adjacente à glândula submandibular esquerda.

Fonte: acervo de fotografias cirúrgicas do Dr. Thiago Calcagnotto.

Observam-se limites pouco definidos entre a lesão lobular e a glândula submandibular. A cirurgia foi realizada sob efeitos de anestesia geral, usando o acesso cirúrgico na região submandibular esquerda por meio de incisão em pele e divulsão por planos até exposição da lesão nodular em glândula, conforme técnica cirúrgica descrita por Ellis ${ }^{16}$. Uma incisão de aproximadamente $10 \mathrm{~cm}$ em pele foi realizada no lado esquerdo, em região cervical submandibular, distante aproximadamente $2 \mathrm{~cm}$ do bordo ósseo mandibular (Figura 2-A). A divulsão tecidual foi realizada por planos até a exposição da glândula submandibular e da lesão nodular adjacente à glândula (Figura 2-B). Observou-se durante a cirurgia que a lesão nodular tinha aspecto superficial irregular e era alimentada por inúmeros vasos sanguíneos que penetravam no seu interior. Os vasos sanguíneos foram ligados por meio de ligadura realizada com fios absorvíveis de poliglactina 4-0, e a lesão nodular foi isolada e removida (Figura 2-C e 2-D). Realizaram-se os cuidados com a ferida operatória, a sutura foi realizada por planos com fio absorvível de poliglactina 4-0 (Figura 2-E) e em pele com Mononylon 6-0 (Figura 2-F). A peça patológica coletada foi imediatamente acomodada em recipiente contendo formalina neutra tamponada a $10 \%$ e enviada para realização de exame histopatológico, a fim de confirmar o diagnóstico clínico de adenoma pleomórfico de glândula submandibular. 


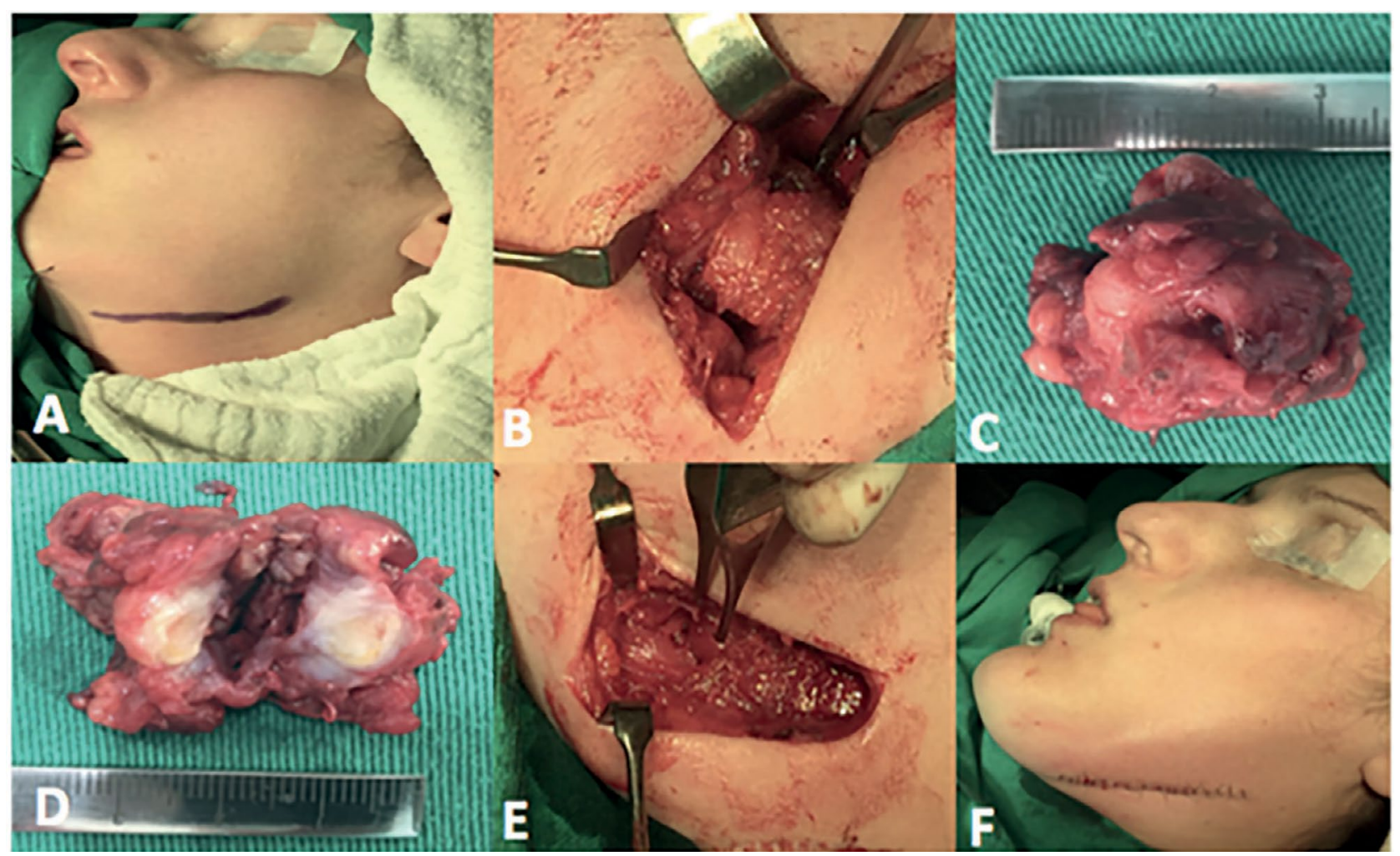

Figura 2 - Imagens transoperatórias

Legenda: demarcação dos limites da incisão submandibular para acesso à glândula (A); acesso por planos e exposição da lesão nodular adjacente à glândula submandibular (B); lesão nodular removida juntamente com a glândula submandibular esquerda (C); aspecto interno da lesão glandular, evidenciando conteúdo sólido (D); sutura interna por planos com fio Vicryl 4-0; e sutura em pele com fio Mononylon 6-0 (F).

Fonte: acervo de fotografias cirúrgicas do Dr. Thiago Calcagnotto.

Após dez dias, o resultado do exame histopatológico chegou ao diagnóstico de adenocarcinoma. A paciente foi orientada sobre o diagnóstico e sobre as condutas em função do diagnóstico e encaminhada para avaliação com oncologista, a fim de seguir tratamento adequado com especialista. A paciente encontra-se em tratamento com especialista, que solicitou, associado a quimioterapia e radioterapia, esvaziamento ganglionar da região cervical por cirurgião de cabeça e pescoço. Ela segue em acompanhamento com o oncologista sem manifestação de crescimento do tumor.

\section{Discussão}

O trabalho teve como objetivo exemplificar, por meio de um caso clínico, aspectos clínicos e imaginológicos e a importância do diagnóstico precoce das neoplasias de glândulas salivares. Desse modo, quando o paciente realizou um exame de ressonância magnética, já havia evidência da ligação da lesão com a glândula salivar. Essa informação poderia levantar a suspeita de uma doença glandular, não somente de um linfonodo infartado, como na hipótese de diagnóstico clínico inicial. Sabe-se que, quanto mais precoce for o diagnóstico, melhor é o prognóstico para o tratamento. Em contrapartida, a paciente aguardou dois anos para buscar a continuidade do acompanhamento, visto que ou não foi orientada a retornar, ou não retornou por vontade própria. A partir disso, tanto a hipótese de diagnóstico como de prognóstico do caso foram alteradas negativamente, uma vez que posteriormente o diagnóstico conclusivo foi de uma lesão maligna ${ }^{13}$.

Depois de dois anos, a paciente retornou para uma nova avaliação, desta vez com um especialista em Cirurgia e Traumatologia Bucomaxilofacial, devido à influência familiar e ao fato de não ser normal tanto tempo uma parte do corpo com tal alteração não sofrer a involução estabelecida no primeiro diagnóstico. Uma possível explicação para essa demora por uma nova procura profissional é o medo do diagnóstico de um câncer, fato que prejudica muito o prognóstico do caso. Por isso fez-se necessário um suporte da equipe médica e odontológica e da família para que a paciente enfrentasse a doença da melhor maneira possível ${ }^{17,18}$.

O medo de um diagnóstico de lesão maligna e a facilidade de aceitação de um quadro clínico mais brando ainda podem justificar a demora em buscar uma nova avaliação. Características visuais e sintomáticas, além de exames complementares, para comprovar a segunda hipótese de diagnóstico e, assim, não negligenciar o quadro clínico e imaginológico da paciente ${ }^{19}$, foram de extrema importância no quesito de obtenção de diagnóstico específico. Tais exames, como ressonância magnética, podem ser 
indicados como forma de obtenção de diagnóstico diferencial, apresentado, numa revisão sistemática, com metanálise que aponta que se alcançou o objetivo pretendido e apresentou semelhanças satisfatórias com a biópsia, além de esse exame ser fornecido no sistema público de saúde brasileiro ${ }^{20}$. Além da ressonância magnética, existem outros exames que também podem ser utilizados, como ultrassonografia e tomografia computorizada, sendo o ultrassom o mais preciso (97,7\%), quando comparado à tomografia computadorizada $(96,1 \%)$, apesar de ambos apresentarem um valor elevado de precisão, segundo estudo comparativo de Mishara (2016) ${ }^{21}$. O ultrassom é amplamente usado, pois é um exame de fácil realização e precisão para avaliação de glândulas e tecidos moles. A imagem no ultrassom de uma glândula salivar acometida por um tumor se apresenta como ecogênica e heterogênea, com bordas irregulares e desfocadas, podendo se continuar com estruturas vizinhas ${ }^{22}$. Outro exame, com finalidade de diagnóstico diferencial, são as biópsias por punção, por meio de punção guiada por ecografia ${ }^{23}$. A partir disso, pode-se observar que, tanto na primeira consulta como na segunda, uma biópsia por punção guiada por ecografia poderia fornecer algum diagnóstico preciso, como o que foi realizado a partir da excisão total da glândula.

A suspeita de um adenoma se deu devido ao não aumento da lesão ao longo do tempo e ao fato de a lesão mostrar-se indolor à pressão leve e com mobilidade, características quase sempre encontradas nesse tipo de tumor ${ }^{24}$.

\section{Considerações finais}

Ressalta-se a importância de se estabelecer um correto diagnóstico por meio da identificação das caraterísticas clínicas e imaginológicas da lesão. Deve-se associar uma entrevista dialogada minuciosa e a confirmação da hipótese de diagnóstico a exames complementes, que são indispensáveis para se chegar ao diagnóstico correto e ao tratamento adequado.

\section{Abstract}

Objective: to address and discuss, through a case report, clinical and imaging aspects capable of establishing the differential diagnosis of tumor lesions of major salivary glands. Case report: the present study discusses, through a case report, the differential diagnosis of major salivary glands in which a patient reports an increase in volume with an initial medical diagnosis of chronic cervical lymphadenopathy caused by a history of recurrent throat infections. Thus, the initial procedure that a head and neck surgeon performed was the proservation of the case. However, after a new reevaluation and complementary examinations requested by the oral and maxillofacial surgery team, due to the insistence of family members, a diagnostic hypothesis of pleomor- phic adenoma of the submandibular gland was made. Consequently, the lesion was accessed and removed by surgery and sent for histopathological diagnosis, which later reached the conclusion of adenocarcinoma of the submandibular gland. Final considerations: the differential diagnosis is essential for determining the choice of treatment of these neoplasms, provided that in addition to the different therapeutic conduct, the malignant lesions may generate metastases in other parts of the body, making the prognosis of survival of the patient less positive. The correct diagnosis associated with adequate therapy and, especially, the early diagnosis of the type of lesion leads to a better quality of life for the patient affected by a malignant neoplasia of major salivary gland.

Keywords: Salivary glands. Differential diagnosis. Adenocarcinoma.

\section{Referências}

1. Fox PC, Ship JA. Salivary gland diesases. In: Greenberg M, Glick M, Ship JA. Burket's Oral Medicine. Hamilton: BC Decker; 2008. p. 191-222.

2. Ord RA, Ghazali N. Margin analysis malignant salivar gland neoplasms of the head and neck. Oral Maxillofac Surg Clin Noth Am 2017; 29(3):315-24.

3. Brookstone MS, Hubos AG, Spiro RH. Central adenoid cystic carcinoma of the mandible. J Oral Maxillofac Surg 1990; 48(12):1329-33.

4. Vargas PA, Gehard R, Filho VJFA, Castro IV. Salivary gland tumors in a Brazilian population: a retrospective study of 124 cases. Rev Hosp Clín Fac Med S Paulo 2002; 57(6):271-6.

5. Yih WY, Kratochvil FJ, Stewart JC. Intraoral minor salivary gland neoplasm: review of 213 cases. J Oral Maxillofac Surg 2005; 63(6):805-10.

6. Santos GC, Martins MR, Pellacani LB, Vieira ACT, Nascimento LA, Abrahão M. Neoplasias de glândulas salivares: estudo de 119 casos. J Bras Patol Med Lab 2003; 39(4):371-5.

7. Guzzo M, Locati LD, Prott FJ, Gatta G, Mcgurk M, Licitra L. Major and minor salvary gland tumors. Crit Rev Oconl Hematol 2010; 74(2):134-48.

8. Matsuba HM, Mauney M, Thawley SE, Simpson JR, Pikul FJ. Adenocarcinomas of major and minor salivary gland origin: a histopathologic review of treatment failure patterns. Laryngoscope 1988; 98(7):784-8.

9. Lee Y-H, Lee JAE-II. Metastatic carcinoma of the oral region: an analysis of 21 cases. Med Oral Patol Oral Cir Bucal 2017; 22(3):359-65.

10. Hashimoto N, Kurihara K, Yamasaki H, Ohba S, Sakai H, Yoshida S. Pathological characteristics of metastatic carcinoma in the human mandible. J Oral Pathol 1987; 16(7):362-7.

11. Irani S. Metastasis to the Jawbones: a review of 453 cases. $\mathrm{J}$ Int Soc Prevent Communit Dent 2017; 7(6):71-81.

12. Li J, Wang BY, Nelson M, Li L, Hu Y, Urken ML, et al. Salivary adenocarcinoma, not otherwise specified: a colletion of orphans. Arch Pathol Lab Med 2014; 128(x):1385-94.

13. Ferrer R. Lynphadenopathy: differential diagnosis and evaluation. Am Fam Physicians 1998; 6(58):1313-20.

14. Seethala RR. Salivary gland tumors: current concepts and controversies. Surg Pathol Clin 2017; 10(1):155-76. 
15. Megwalu U, Sirjani D. Risk of nodal metastasis in major salivary gland adenoid cystic carcinoma. Otolaryngol Head Neck Surg 2017; 146(4):660-4.

16. Ellis IIIE, Zide MF. Acessos transfaciais para mandíbula. In: Ellis IIIE, Zide MF. Acesso cirúrgico ao esqueleto facial. 2. ed. Santos: Editora Santos; 2006. p. 151.

17. Marlow LAV, Waller J, Wardle J. Barriers to cervical cancer screening among ethnic minority women: a qualitative study. J Fam Plann Reprod Health Care 2015; 41(4):248-54.

18. Lehto US, Aromaa A, Tammela TL. Experiences and psychological distress of spouses of prostate cancer patients at time of diagnosis and primary treatment. Eur J Cancer Care (Engl.) 2017; e12729: 1-11.

19. Ogden G. Early detection of oral cancer: how do i ensure i don't miss a tumour? Dent. Uptade 2013; 40(6):462-5.

20. Moreira MA, Lessa LS, Bortoli FR, Lopes A, Xavier EPX, Ceretta RA et al. Meta-analysis of magnectic resonance imaging accuracy for diagnosis of oral cancer. Plos One 2017; 12(5):1-15.

21. Mishara N, Rath KC, Upadhyay UN, Raut S, Baig SA, Birmiwal KG. Preoperative evaluation of cervical lymph nodes for metastasis in patients with oral squamous cell carcinoma: a comparative study of efficacy of palpation, ultrasonography and computes tomography. Natl J Maxilloafac Surg 2016; 7(2):186-90.

22. Zajkowski P, Ochal-Choinska A. Standards for the assessment of salivar glands - an update. J Ultrason 2016; 16(65):175-90.

23. Bobati SS, Patil BV, Dombale VD. Histopathological study of salivary gland tumors. J Oral Maxillofac Pathol 2017; 21(1):46-50.

24. Patil P. Pleomorphic adenoma of submandibular gland: a case report with review of literature. Dent. Res. J. 2017; 11(3):411-14.

Endereço para correspondência:

Thiago Calcagnotto

Rua São João, 942, Centro

93010-250, São Leopoldo, RS, Brasil

Telefone: +51-54-99174-6104

E-mail: tcalcagnotto@hotmail.com

Recebido: 29/06/2017. Aceito: 29/07/2017. 